\title{
A Check of a $D=4$ Field-Theoretical Calculation Using the High-Temperature Expansion for Dyson's Hierarchical Model
}

\author{
J. J. Godina \\ Dep. de Fis. , CINVESTAV-IPN, Ap. Post. 14-740, Mexico, D.F. 07000 \\ and \\ Dpt. of Physics and Astr., Univ. of Iowa, Iowa City, Iowa 52246, USA \\ Y. Meurice日 \\ CERN, 1211 Geneva 23, Switzerland \\ and \\ Dpt. of Physics and Astr., Univ. of Iowa, Iowa City, Iowa 52246, USA \\ S. Niermann \\ Dpt. of Physics and Astr., Univ. of Iowa, Iowa City, Iowa 52246, USA
}

\begin{abstract}
We calculate the high-temperature expansion of the 2-point function up to order 800 in $\beta$. We show that estimations of the critical exponent $\gamma$ based on asymptotic analysis are not very accurate in presence of confluent logarithmic singularities. Using a direct comparison between the actual series and the series obtained from a parametrization of the form $\left.\left(\beta_{c}-\beta\right)^{-\gamma}\left(\operatorname{Ln}\left(\beta_{c}-\beta\right)\right)^{p}+r\right)$, we show that the errors are minimized for $\gamma=0.9997$ and $p=0.3351$, in very good agreement with field-theoretical calculations. We briefly discuss the related questions of triviality and hyperscaling.
\end{abstract}




\section{INTRODUCTION}

The dimension four plays a doubly important role in physics. First, it is the dimension of space-time which is relevant for a relativistic description of a large class of phenomena, from electricity and magnetism to scattering processes at the highest experimentally accessible energies. Second, it is the upper critical dimension for scalar field theory. If one analytically continues the renormalization group equations [1] (usually derived within some approximation) to non-integer dimensions, it appears that when the dimension tends to four from below, the non-trivial fixed point merges with the Gaussian one. This justifies the $\epsilon$-expansion.

It is thus commonly accepted that in four dimensions, the critical exponents are the trivial ones (i.e. those obtained from mean field). Unfortunately, it often difficult to find clear evidence for or against trivial exponents, for instance, from high-temperature (HT) series [2,3] or a finite volume calculation [4]. The root of the problem is the existence of a marginal direction which makes the approach to the fixed point more intricated than in three dimensions. The corrections to the power laws can in principle be obtained from the Callan-Symanzik equations, provided we know the exact form of the various functions (beta, gamma, ... ) entering into them. Using the lowest order in perturbation theory, Brezin, Le Guillou, and Zinn-Justin [5] found that the trivial power divergences get multiplied by rational powers of $\operatorname{Ln}\left(\beta_{c}-\beta\right)$. It is important to check this result with methods independent of perturbation theory. In particular, it is conceivable that there exist non-trivial fixed points which cannot be revealed by perturbation theory.

The technical challenge which appears in any kind of calculation is to distinguish between a small change (with respect to the trivial value) in the critical exponent and a slowly varying (compared to the trivial singularity) multiplicative change. This difficulty appears clearly in the asymptotic analysis of the high-temperature expansion of the susceptibility, where the leading term of the extrapolated slope defined in Eq. (3.4) $(\gamma-1)$ can be small compared to corrections proportional to the inverse of the logarithm of the order, unless one can reach an astronomically large order.

Another interesting feature of the field-theoretical method is the so-called hyperscaling relation among the power singularities of the 2- and 4-point (subtracted) Green's functions at zero momentum. In three dimensions, the violations of hyperscaling [6] are hard to resolve by high-temperature calculation. This is still a controversial [7] topic. In four dimensions, conflicting [2, 3] conclusions were drawn from the high-temperature series.

The confirmation of the field-theoretical results would require that an unbiased estimate of the main power singularity and the power of the logarithmic correction come close to their predicted values, with errors compatible with (small) higher-order corrections. We propose here to test the field-theoretical results using an expansion in the kinetic term (also called high-temperature expansion), in a model which is obviously non-trivial in three dimensions, but where calculations are easier than in nearest-neighbor lattice models.

The hierarchical model [8] is a non-trivial approximation of models with short range interactions, which is well-studied [9, [10], and for which we can calculate the high-temperature expansion [11 to a very large order. The recursion relation which summarizes the renormalization group transformation of this model is closely related to the approximate recursion formula discussed by Wilson [1]. The qualitative and quantitative aspects of this relationship 
are discussed in Ref. [12].

In recent publications [13,14, we reported results concerning the high-temperature expansion of Dyson's hierarchical model in three dimensions. We calculated the hightemperature expansion of the magnetic susceptibility up to order 800 with Ising and LandauGinzburg measures. This allowed us to obtain a value [14] of the critical exponent $\gamma$ of 1.300 in $D=3$, with estimated errors of order 0.002 . This result is consistent with the results obtained with the $\epsilon$-expansion [9, 10].

We found clear evidence for oscillations in the quantity, called the extrapolated slope 15] (see section below), used to estimate the critical exponent $\gamma$. When using a log scale for the order in the high-temperature expansion, these oscillations become regularly spaced. Our interpretation of the data was consistent with the hypothesis that the eigenvalues of the linearized renormalization group transformation are real, but that the constants appearing in the conventional parametrization of the magnetic susceptibility should be replaced by functions of $\beta_{c}-\beta$ invariant under the rescaling of $\beta_{c}-\beta$ by $\lambda_{1}$, the largest eigenvalue of the linearized renormalization group transformation. This possibility has been mentioned in the past by K. Wilson [四] and developed systematically by Niemeijer and van Leeuwen [16]. Our analysis provided good evidence that the oscillations appear with a universal frequency in good agreement with theoretical expectations, but with a measure-dependent phase and amplitude.

Subsequently, more efficient methods of calculation, based on finite dimensional projections of the Fourier transform of the recursion formula, were developed. As explained in detail in Ref. [17], the effects of such truncations can be controlled with a precision which is better than exponential when the dimension of the truncated space increases.

In this paper, we study the high-temperature expansion of Dyson's hierarchical model in dimension 4. For the sake of completeness, we briefly review the method of calculation in section [1]. The conventional methods [15,18 used to estimate the critical temperature and a critical exponent from a high-temperature series are reviewed in section III. We show that in the presence of logarithmic corrections to the scaling laws, the asymptotic behavior of the corrections is modified. The extrapolated ratio defined in Eq. (3.3) provides an estimate of the critical temperature with corrections of order $m^{-1} \times(L n(m))^{-2}$, where $m$ is the order in the high-temperature expansion. In the following, we continue to use the notation $m$ with the same meaning. Using the expansion of the susceptibility up to order 800 , we obtained a value of the critical temperature which agreed with the high-precision determination of Ref. 17] with errors of less than one part in 10,000. On the other hand, the extrapolated slope defined in Eq. (2) estimates the critical exponent minus one with corrections which are only suppressed by $(\operatorname{Ln}(m))^{-1}$. If this weak suppression is not recognized, one may conclude that the critical exponent $\gamma$ takes a value larger than the trivial one. More generally, asymptotic analysis is not adequate to distinguish between a value of $\gamma$ close to 1 and a correction to the scaling laws which is less singular than a power.

In section IV, we analyze the high-temperature expansion of the susceptibility without relying on the asymptotic behavior of the coefficients. We use $h(m) \equiv\left(r_{m} \beta_{c}-1\right) m$, a function which represents the difference between the ratio of successive coefficients $r_{m}$ and its asymptotic value $\beta_{c}^{-1}$. The function $h(m)$ can be calculated exactly using either the empirical series or the series corresponding to a given assumption on the analytical form of the susceptibility. Taking the sum over a large range of $m$ of the square of the differences 
between these two values of $(h(m))^{-1}$, one can get an error function which indicates how good the analytical assumption is. We found that the parametrization

$$
\chi=\left(\beta_{c}-\beta\right)^{-\gamma}\left(A_{0}\left(-\beta^{-1} \operatorname{Ln}\left(1-\frac{\beta}{\beta_{c}}\right)\right)^{p}+A_{1}\right)
$$

provides very good fits of the data for $\gamma \simeq 1$ and $p \simeq \frac{1}{3}$, which is the field-theoretical [5] result. In order to decide how accurate the agreement is, we have considered fixed values of $\gamma$ in the vicinity of 1 and equally spaced by $10^{-4}$ steps. For each of these values, we have determined the values of $p$ and $A_{1} / A_{0}$ which minimize the error function. This error function behaves like a paraboloid near its minimum at $\gamma=0.9997$ and $p=0.3351$, in good agreement with the field-theoretical calculation. The errors on this estimate are mostly systematic. To get more accurate results, one needs to replace the constant $A_{1}$ by a slowly varying function.

Another quantity which can be studied using the high-temperature expansion is the dimensionless renormalized coupling constant [19], denoted $\lambda_{4}$ hereafter, obtained by multiplying the connected four-point function at zero momentum by the eighth $(D+4$ in general) power of the renormalized mass. For $D<4$, this quantity is designed to have a finite and non-zero limit when $\beta \rightarrow \beta_{c}$. In the case $D=4$, we have checked with good accuracy [17] that $\lambda_{4}$ goes to zero like $\left(\operatorname{Ln}\left(\beta_{c}-\beta\right)\right)^{-1}$ for the model studied here. The calculation of the HT coefficients of $\lambda_{4}$ involves the subtraction of the disconnected part and suffers the same type of numerical problems as the direct calculation of $\lambda_{4}$, as discussed in Ref. [17]. For this reason, we were only able to extract a series of 30 coefficients. The analysis of this series is consistent with the fact that $\lambda_{4}$ goes to zero when $\beta \rightarrow \beta_{c}$ (triviality), but it is not possible to distinguish a $\left(\operatorname{Ln}\left(\beta_{c}-\beta\right)\right)^{-1}$ approach to zero from a $\left(\beta_{c}-\beta\right)^{1 / 2}$ approach, which would be necessary to establish whether or not hyperscaling holds. This question has been settled in Ref. [17], and this section illustrates the inconclusiveness of results obtained from short series.

In conclusion, we have shown that by using sufficiently long series and methods of analysis not relying on an asymptotic expansion, it is possible to obtain very good agreement between calculations based on field theory and those based on high-temperature expansion in the upper critical dimension. We emphasize that the main interest of the high-temperature expansion is to allow us to probe global features of the renormalization group flows which cannot be approached using renormalized perturbation theory or an analysis of the linearized behavior near the fixed point. An example of such a global feature is the existence of logperiodic oscillations 13, 14, which play an important role in $D=3$, but have an almost negligible effect in $D=4$, as shown in section IIII. Another example of a global feature could be the existence of a non-trivial fixed point. The good agreement found in section IV makes this possibility very implausible for the model studied here.

\section{CALCULATIONS OF THE HT COEFFiCIENTS}

The calculation of the high-temperature expansion of the unsubtracted $2 k$-point functions of Dyson's hierarchical model can be performed iteratively using the basic recursion formula in its Fourier form [11. This method has been discussed extensively in Refs. 13, 14. For 
the sake of being self-contained, we briefly explain the basic method of calculation. More details, justifications, and motivations can be found in Refs. [4, 13, 14.

The recursion formula for the rescaled Fourier transform $R_{n}(k)$ of the local measure for blocks of $2^{n}$ sites reads

$$
R_{n+1}(k)=C_{n+1} \exp \left(-\frac{1}{2} \beta\left(\frac{c}{4} s^{2}\right)^{n+1} \frac{\partial^{2}}{\partial k^{2}}\right)\left(R_{n}\left(\frac{k}{s}\right)\right)^{2},
$$

where $c$ is an adjustable parameter which takes the value $2^{1-\frac{1}{D}}$, in order to approximate $D$-dimensional models. In the following, we will only consider the case $D=4$, which means $c=\sqrt{2}$. The rescaling operation commutes with iterative integrations, and the rescaling factor $s$ can be fixed at our convenience. In order to obtain stabilized expressions in the high-temperature phase, we will take $s=\sqrt{2}$ in the following. We fix the normalization constant $C_{n}$ in such way that $R_{n}(0)=1 . R_{n}(k)$ then has a direct probabilistic interpretation. If we call $M_{n}$ the total field $\sum \phi_{x}$ inside blocks of side $2^{n}$, and $<\ldots>_{n}$ the average calculated without taking into account the interactions among these blocks, we can write

$$
R_{n}(k)=\sum_{q=0}^{\infty} \frac{(-i k)^{2 q}}{2 q !} \frac{\left(M_{n}\right)^{2 q}>_{n}}{2^{q n}} .
$$

We see that the Fourier transform of the local measure obtained after $n$ iterations generates the zero-momentum Green's functions calculated with $2^{n}$ sites. All the calculations done here use an initial Ising measure, which means that $R_{0}(k)=\cos (k)$. Since we are interested in the leading singularity, this choice should play no role [19] in the discussion.

The high-temperature expansion of the zero-momentum Green's function can be obtained from an expansion of Eq. (2.1) in powers of $\beta$. The most important sources of errors are the round-off errors. After 100 iterations, the relative errors on the $m$ th coefficient [14] are of the order of $m \times 10^{-15}$. With the choice $s=\sqrt{2}$, the coefficients reach a finite value in the infinite volume limit. Actual computations are made at large but finite volume (i. e. at finite $n$ ). The relative difference between the coefficients at finite and infinite $n$ goes to zero 11] like $\left(\frac{c}{2}\right)^{n}$. For $D=4$, the choice $n=100$ means that $\left(\frac{c}{2}\right)^{n}=2^{-50}$, which is smaller than the numerical errors.

Such a calculation is in general time-consuming when one wants to calculate more than 100 coefficients. It is, however, possible to save time by using finite dimensional approximations [17] of degree $l$ for the generating function:

$$
R_{n}(k)=1+a_{n, 1} k^{2}+a_{n, 2} k^{4}+\ldots .+a_{n, l} k^{2 l},
$$

with $l$ much smaller than the required dimension $m+1$ necessary for an exact [11] calculation. After each iteration, non-zero coefficients of higher order $\left(a_{n+1, l+1}\right.$ etc. $)$ are obtained, but set to zero in the next iteration. The $l$-dependence of the high-temperature coefficients of the susceptibility is discussed in Ref. [17]. If $b_{m}^{(l)}$ denotes the value of $b_{m}$ in a truncated space of dimension $l$, we found that

$$
b_{m}^{(l)}=b_{m}\left(1-l^{-|s| l+q}\right)
$$

where $s$ and $i$ are, respectively, the slope and intercept of the corresponding fitted line, as shown in Fig. 1. The intercepts are approximately 2.3, while the slopes depend on $m$. 
Eq. (2.4) represents suppressions which are better than exponential. From this figure, we can check, for instance, that for $m=400$ (which is the maximal value used in section IV), the extrapolated errors at $l=40$ are significantly lower than the numerical errors. Using extrapolation in $m$, it was estimated in Ref. [17] that in the case $D=4, l=38$ was sufficient to calculate $b_{1000}$.

In summary, the following calculations will be performed with $l=50$ and $n=100$. The above discussion shows that this choice guarantees that the systematic errors are smaller than the numerical errors.

\section{THE LIMITATION OF THE ASYMPTOTIC ANALYSIS IN PRESENCE OF CONFLUENT LOGARITHMIC SINGULARITIES}

In this section, we study the singularities of the susceptibility using its high-temperature expansion

$$
\chi(\beta)=\sum_{m=0}^{\infty} b_{m} \beta^{m} .
$$

We define $r_{m}=b_{m} / b_{m-1}$, the ratio of two successive coefficients. When $D<4$, one expects |19 that

$$
\chi=\left(\beta_{c}-\beta\right)^{-\gamma}\left(A_{0}+A_{1}\left(\beta_{c}-\beta\right)^{\Delta}+\ldots\right),
$$

and it is convenient to introduce quantities [15] called the extrapolated ratio $\left(\widehat{R}_{m}\right)$ and the extrapolated slope $\left(\widehat{S}_{m}\right)$ in order to estimate $\beta_{c}$ and $\gamma$. These quantities are defined as

$$
\widehat{R}_{m}=m r_{m}-(m-1) r_{m-1}
$$

and

$$
\widehat{S}_{m}=m S_{m}-(m-1) S_{m-1}
$$

where

$$
S_{m}=-m(m-1)\left(r_{m}-r_{m-1}\right) /\left(m r_{m}-(m-1) r_{m-1}\right)
$$

is called the normalized slope. When $A_{0}$ and $A_{1}$ are constant, one finds 15 that the $1 / \mathrm{m}$ corrections disappear:

$$
\widehat{S}_{m}=\gamma-1-B m^{-\Delta}+O\left(m^{-2}\right) .
$$

However, for the hierarchical model in $D=3$, large oscillations were observed [13] in $\widehat{S}_{m}$ and it was recognized 13,14 that $A_{0}$ and $A_{1}$ should be considered as functions of $\beta_{c}-\beta$ invariant under the rescaling of $\beta_{c}-\beta$ by $\lambda_{1}$, the largest eigenvalue of the linearized renormalization group transformation. The asymptotic analysis (when $m$ becomes large) of the extrapolated slope in this modified situation is given in section 3 of Ref. [14. It was found that $1 / m$ corrections with rather large coefficients reappeared. Nevertheless, it was possible to extract the critical exponent $\gamma$ with estimated errors of 0.2 percent. 
The situation is very different in $D=4$, as shown in Fig. 2. The oscillations are barely visible for low values of $m$, and not visible at all for larger $m$, where $\widehat{S}_{m}$ appears to decay smoothly. If the parametrization of Eq. (3.2) and its corollary Eq. (3.6) applied, one might conclude that $\gamma$ is close to 1.05. However, if we plot the inverse of $\left(S_{m}\right)^{-1}$ versus $\operatorname{Ln}(m)$, we find the linear behavior shown in Fig. 3. This shows that $\widehat{S}_{m}$ decays like $1 / \operatorname{Ln}(m)$, so Eq. (3.6) does not provide an adequate description of the situation. The deviation from the linear behavior shows an interesting fine structure shown in Fig. 因. For $m$ near $400(\operatorname{Ln}(m)$ near 6), ones sees that the amplitude of oscillation is almost four orders of magnitude smaller than $\left(\widehat{S}_{m}\right)^{-1}$ itself. For such a values of $m$, the numerical errors become comparable with the oscillations. For larger values of $m$, the numerical errors become larger and wash out the oscillations. The numerical errors on $\left(\widehat{S}_{m}\right)^{-1}$ in $D=4$ are of the same order of magnitude as what we would estimate in $D=3$ from the error analysis of Ref. [14]. The main difference is that the oscillations have a much smaller amplitude in $D=4$. In the following, we will treat the oscillations on the same footing as the numerical errors, which is justified for $m$ sufficiently large.

We will now revisit the asymptotic analysis of $\widehat{R}_{m}$ and $\widehat{S}_{m}$ in a more general case than Eq. (3.2) with $A_{0}$ and $A_{1}$ constant. Our main assumption will be that

$$
\chi(\beta)=\left(1-\frac{\beta}{\beta_{c}}\right)^{-\gamma} G\left(1-\frac{\beta}{\beta_{c}}\right),
$$

where $G$ is such that

$$
\lim _{m \rightarrow \infty} \frac{G^{\prime}\left(\frac{\gamma}{m}\right)}{m G\left(\frac{\gamma}{m}\right)}=0 .
$$

This restriction includes the case where $G\left(1-\frac{\beta}{\beta_{c}}\right)$ grows like a positive power of a logarithm when $\beta$ goes to $\beta_{c}$. We then proceed as in ref. [18] and explain the principle of the asymptotic expansion. We use the residue theorem in the complex $\beta$ plane to get an integral representation of the coefficients. Next we treat the integral with the steepest descent method. Using an exponential parametrization for the integrand of the $m$ th coefficient, one finds that the phase has a maximum for a value of $y=\frac{\beta}{\beta_{c}}$ such that

$$
y\left(\frac{\gamma}{1-y}-\frac{G^{\prime}(1-y)}{G(1-y)}\right)=m+1 .
$$

The basic principle of calculation is that the second term of the l. h. s. of this equation can be treated as a perturbation, for $m$ sufficiently large. Neglecting this second term, we get

$$
y=1-\frac{\gamma}{m}+O\left(\frac{1}{m^{2}}\right) .
$$

Eq. (3.8) is then seen as the condition which allows us to treat the second term of the 1 . h. s. of Eq. (3.10) as a perturbation. Finally, one finds [18] that for large $m$, the leading contribution to the $m$ th coefficient has the form

$$
b_{m} \propto \beta_{c}^{-m} m^{\gamma-1} G\left(\frac{\gamma}{m}\right) .
$$


Before going further, we introduce a parametrization of the ratio of successive coefficients:

$$
r_{m}=\left(\frac{1}{\beta_{c}}\right)\left(1+\frac{1}{m} h(m)\right) .
$$

This definition is independent of any kind of expansion. From Eq. 3.11, we found the asymptotic estimate

$$
h(m)=\left(\gamma-1-\frac{\gamma}{m} \frac{G^{\prime}\left(\frac{\gamma}{m}\right)}{G\left(\frac{\gamma}{m}\right)}\right)+\ldots
$$

If we consider the case

$$
G(x) \propto(-\operatorname{Ln}(x))^{p}
$$

we obtain

$$
\widehat{R}_{m}=\left(\frac{1}{\beta_{c}}\right)\left(1+O\left(\frac{1}{m(\operatorname{Ln}(m))^{2}}\right)\right)
$$

and

$$
\widehat{S}_{m}=\gamma-1+O\left(\frac{1}{\operatorname{Ln}(m)}\right) .
$$

From this, we can conclude that under the assumption of Eq. (3.14), asymptotic analysis justifies using $\widehat{R}_{m}$ as an estimator for $\frac{1}{\beta_{c}}$, with estimated errors on the order of $10^{-4}$. This quantity is displayed in Fig. 5. As expected, no oscillations are visible. The change between $m=200$ and $m=800$ is less than $10^{-4}$, which is consistent with $\frac{1}{m(\operatorname{Ln}(m))^{2}}$ corrections. If we use $\widehat{R}_{800}$ as our best estimate, we obtain $\beta_{c}=0.665548$, which is in good agreement with our accurate calculation [17], where we found $\beta_{c}=0.6654955715318593$. The discrepancy has the same order of magnitude as the small variations noted above.

On the other hand, for $\widehat{S}_{m}$, the corrections to $\gamma-1$ are not very small. For instance, for $m=800,(\operatorname{Ln}(m))^{-1} \simeq 0.15$, and it seems implausible that one could establish that $|\gamma-1|<10^{-3}$ on the grounds of an expansion in this not-very small parameter. More generally, it takes exponentially large $m$ for the "corrections" in $(\operatorname{Ln}(m))^{-1}$ to become smaller than the "leading" $\gamma-1$ when this quantity is small. Thus it seems desirable to use non-asymptotic methods, the subject of the next section.

\section{A DIRECT ESTIMATION OF THE CRITICAL EXPONENTS}

In this section, we propose to use direct calculations of $h(m)$, defined as

$$
h(m)=\left(r_{m} \beta_{c}-1\right) m .
$$

This quantity can be calculated exactly under some assumption regarding the susceptibility, and calculated exactly from the empirical series. We emphasize that $h(m)$ is defined from Eq. (3.12) and its calculation does not require any kind of expansion. However, we need to 
provide an estimate of $\beta_{c}$. In the following, we will take the most accurate value [17] of $\beta_{c}$ quoted in the previous section rather than the approximate values obtained from $\widehat{R}_{m}$.

A simple assumption on the leading singularity of the susceptibility in $D=4$ is given by the result of a field-theoretical calculation [0]

$$
\chi=\left(\beta_{c}-\beta\right)^{-1}\left(A_{0}\left(\left|\ln \left(\beta_{c}-\beta\right)\right|\right)^{\frac{1}{3}}\right) .
$$

This lowest-order result would also be obtained for Dyson's model, because at this order, numerical factors (integrations over the angles), which are model-dependent, cancel. Given that $r_{m}$ is the ratio of two successive coefficients, it is independent of $A_{0}$ and it transform as $r_{m} \rightarrow r_{m} s^{-1}$ under a rescaling $\beta \rightarrow s \beta$. Consequently, $r_{m} \beta_{c}$ is independent of the choice of $A_{0}$ and $\beta_{c}$. We have thus calculated $h(m)$ from the expansion of

$$
f(x)=(1-x)^{-1}\left(-\frac{\ln (1-x)}{x}\right)^{\frac{1}{3}}
$$

in $x$, about $x=0$. The variable $x$ stands for $\beta / \beta_{c}$. The division by $x$ does not change the leading singularity [3] when $x \rightarrow 1$ while providing a regular expansion around $x=0$. Under the assumption of Eq.(4.2), we find from Eq.(3.13) that asymptotically $h(m)$ tends to a small and possibly zero constant plus a correction which decays like $\frac{1}{\operatorname{Ln}(m)}$. It is thus natural to plot $(h(m))^{-1}$ versus $\operatorname{Ln}(m)$. Such a plot is provided in Fig. 6, where we compare with $(h(m))^{-1}$ calculated directly from the $D=4 \mathrm{HT}$ series, using the definition Eq. (4.1). The two (approximate) lines are separated by an almost constant gap. We tried to modify the assumption Eq. (4.3) in such way that the two lines coincide. The only satisfactory solution we found was the modified assumption

$$
f(x)=(1-x)^{-1}\left(\left(-\frac{\ln (1-x)}{x}\right)^{\frac{1}{3}}+r\right),
$$

where $r$ has to be determined by an error-minimization procedure which we now proceed to explain.

For notational purposes, we call $t(m)$ the "true" value of $(h(m))^{-1}$ obtained from the HT series and $a(m)$ the value of $(h(m))^{-1}$ corresponding to an assumed series such as the one obtained from Eq. (4.4). In practical calculations, we have used the instruction Series in Mathematica to calculate $a(m)$. It should be noted, that for large orders, rational values of the exponents give better numerical results. In addition, if the denominator of this rational exponent gets too large (typically $10^{7}$ for a calculation up to order 400), one runs out of memory. This procedure is quite time-consuming when one goes to large order. Since such a calculation will have to be repeated many times in the rest of this section, we have used the region $300 \leq m \leq 400$ to evaluate the discrepancy between $a(m)$ and $t(m)$. As we can see from the discussion of section [II], in this range the oscillations are already small and

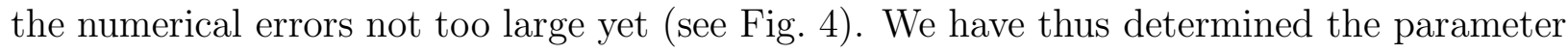
$r$ in Eq. (4.4) by minimizing

$$
E=\sum_{m=300}^{m=400}(t(m)-a(m))^{2} .
$$

The values of $E$ for values of $r$ separated by 0.001 are shown in Fig. 7). The curve can be fitted very well by a parabola. The minimum of this parabola is then determined analytically 
from the three values defining the fitting parabola. This allows us to find the value of $r$ with a precision of $10^{-6}$, three orders of magnitude smaller than the original resolution. The value of $r$ minimizing $E$ found with this procedure is -0.435622 , corresponding to a value of $E$ of order $10^{-4}$. Subsequently, we checked this answer by repeating the calculation of $E$ with a resolution $10^{-6}$ in $r$ and found the same answer. For this value of $r, t(m)$ and $a(m)$ cannot be distinguished in a graph like Fig. 6. The difference between $a(m)$ and $t(m)$ is shown in Fig. 8. In the region where the fit was performed $(300 \leq m \leq 400)$, the differences are 4 orders of magnitude smaller than the values themselves.

We conclude from this analysis that Eq. (4.4) is a very good guess concerning the leading and subleading singularities of the susceptibility. However, we would like to see if it remains the best guess if we allow the exponents to change. In other words, we would to see if different values of the exponents could also be acceptable from the point of view of the high-temperature expansion. We have thus considered a more general assumption:

$$
f(x)=(1-x)^{-\gamma}\left(\left(-\frac{\ln (1-x)}{x}\right)^{p}+r\right)
$$

and studied $E$ as a function of $\gamma, p$, and $r$.

Near a minimum, $E$ behaves generically as a three-dimensional paraboloid. In this region, one can "eliminate" $r$ by fixing its value in such a way that $E$ is minimized with $\gamma$ and $p$ kept constant. The variable $r$ is thus replaced by a linear combination of $\gamma$ and $p$ plus a constant and we can then work with a two-dimensional paraboloid. A section of this paraboloid defined by the condition $\gamma=1$ is shown in Fig. 9. In a second step, one can similarly eliminate $p$ with $\gamma$ fixed by requiring that it takes the $\gamma$-dependent value that minimize $E$. In the case $\gamma=1$ illustrated in Fig. 9, this value of $p$ is 0.32775 , not far from the expected [5] value $\frac{1}{3}$. This show incidentally that a biased estimate of $p$ is in good agreement with the field-theoretical result. Taking values of $\gamma$ separated by $10^{-4}$, we have similarly calculated the value of $p$ given by the minimization condition. The results are shown in Fig. 10. The linear behavior was expected: since near the minimum $E$ is a quadratic form, the minimization condition is linear. Using this linear relation to eliminate $p, E(\gamma)$ becomes a parabola. The minimum value taken by this function is then the minimum of the initial function $E(\gamma, p, r)$. This function is shown in Fig. 11. $E$ is minimized for $\gamma=0.9997$, which according to Fig. 10 corresponds to a value of $p$ of 0.3351 .

In practical calculations, it is convenient to replace parabolic fits by successive applications of Newton's method. This method has an adjustable resolution and it allows one to start in regions away from the minimum, and where the parabolic behavior does not necessarily hold.

It is difficult to estimate the errors on our result. Since the parabolas shown above are reasonably smooth, it seems unlikely that the numerical errors or the oscillations, which should have about the same size, play any significant role. Most likely, the main source of error is that $r$ has been considered as a constant. If instead we allow $r$ to be a slowly varying function of $\beta$, we expect in a model independent way, that these slow variations in $\beta$ will induce slow variations in $m$ of the quantity $(h(m))^{-1}$. In the interval of $m$ considered for the calculation of $E$, the slow variations can be approximated with polynomials. In order to get an idea about how low $E$ could become under such a circumstances, we have fitted the differences between $t(m)$ and $a(m)$ displayed in Fig. 8 and calculated the value 
of $E$ obtained after subtracting these fits from the original differences. For a linear fit, we obtained $E=6 \times 10^{-8}$ and for a quadratic fit $E=8 \times 10^{-10}$. This shows that by keeping $\gamma=1$ and $p=1 / 3$ and allowing $r$ to to be a slowly changing function written in terms of parameters which are adjusted to minimize $E$, we can obtain values of $E$ comparable to those obtained by keeping $r$ as a constant and allowing $\gamma, p$ and $r$ to be adjusted in order to minimize $E$. For definiteness, with $\gamma=1$ and $p$ and $r$ varied to minimize $E$, we obtain $E=6 \times 10^{-9}$. Varying $\gamma, p$ and $r$, we obtain $E=4 \times 10^{-10}$. In conclusion, if we want a more precise estimation of the critical exponents, we also need more information regarding the $\beta$-dependence of the subleading singularities.

\section{TRIVIALITY AND HYPERSCALING}

Another quantity which can be studied using the high-temperature expansion is the dimensionless renormalized coupling constant [19]

$$
\lambda_{4}=-G_{4}^{c} m_{R}^{D+4}
$$

where $G_{4}^{c}$ is the the zero-momentum connected Green's function and $m_{R}$ the renormalized mass. For $D<4$, this quantity is designed to have a finite limit when $\beta \rightarrow \beta_{c}$. In the case $D=3$, we have checked [17] by a direct calcualtion that $\lambda_{4}$ reaches the value 1.92786 when $\beta \rightarrow \beta_{c}$. In the case $D=4$, we have checked with a good accuracy that, in the same limit, $\lambda_{4}$ goes to zero like $\left(\operatorname{Ln}\left(\beta_{c}-\beta\right)\right)^{-1}$. Thus we have direct evidence that in these two cases the power singularities cancel in Eq. (5.1) - in other words, that hyperscaling holds.

Bearing in mind that there is no wave function renormalization $(\eta=0)$ in the hierarchical model, we will define as in Ref. [17] that $\lambda_{4}$ is the limit where $n \rightarrow \infty$ of

$$
\lambda_{4, n}=\frac{<M_{n}^{4}>_{n}-3\left(<M_{n}^{2}>_{n}\right)^{2}}{2^{n}\left(\frac{<M_{n}^{2}>_{n}}{2^{n}}\right)^{\frac{D}{2}+2}},
$$

with the same notation as in Eq. (2.2). Equivalently, with the rescaling factor fixed to $s=\sqrt{2}$ in Eq. (2.1) and the convention of Eq. (2.3),

$$
\lambda_{4, n}=12 \frac{a_{n, 1}^{2}-2 a_{n, 2}}{\left(-2 a_{n, 1}\right)^{\frac{D}{2}+2}} 2^{n} .
$$

The calculation of the HT coefficients of $\lambda_{4}$ involves the subtraction of the disconnected part and it suffers the same type of numerical problems as the direct calculation of $\lambda_{4}$, as discussed in Ref. [17]. For this reason, we were only able to extract a series of 30 coefficients. The quantity $h(m)$ defined in Eq. (4.1) corresponding to this series is displayed in Fig. 12. The figure indicates that this quantity has damped oscillations. The average value of $h(m)$ in the displayed interval is -1.4. From Eq. (3.13), this is consistent with the fact that $\lambda_{4}$ has a finite limit when $\beta \rightarrow \beta_{c}$, but it not possible to distinguish a $\left(\operatorname{Ln}\left(\beta_{c}-\beta\right)\right)^{-1}$ approach to zero from a $\left(\beta_{c}-\beta\right)^{1 / 2}$ approach. For comparison, we have displayed in Fig. 12 the function $h(m)$ corresponding to the series generated by $-x / \operatorname{Ln}(1-x)$ and $(1-x)^{\frac{1}{2}}, x$ being short for $\frac{\beta}{\beta_{c}}$. It is clear that the oscillations make the discrimination between these two asumptions impossible. 
Another way of seeing that the series is too short to describe the details of the behavior near $\beta_{c}$ is to plot the truncated expansion of $\lambda_{4}$ up to order 30. This is done in Fig. 13. The HT expansion indicates correctly that $\lambda_{4}$ goes to zero when $\beta$ increases. However, the behavior near $\beta_{c}$ is not accurate. For comparison, Fig. 13 also shows the leading critical behavior estimated in Ref. [17], namely

$$
\lambda_{4} \simeq \frac{1}{-1.955-0.746 \times \operatorname{Ln}\left(\beta_{c}-\beta\right)} .
$$

The data interpolates nicely between the two types of behavior, but we see that there is no region in the figure where they overlap. The order $30 \mathrm{HT}$ expansion gives accurate results for $\beta_{c}-\beta>3 \times 10^{-2}$, while Eq. (5.4) becomes accurate when $\beta_{c}-\beta<10^{-3}$.

In summary, the truncated expansion makes clear that $\lambda_{4}$ goes to zero when $\beta \rightarrow \beta_{c}$. In other words, the theory is trivial. However, the series is too short to extract accurately the precise way it approaches zero, and one cannot decide from this information whether or not hyperscaling holds.

\section{CONCLUSIONS}

There have been questions [6] in the past regarding possible discrepancies between fieldtheoretical calculations based on the renormalization group approach and calculations based on the high-temperature expansion. Using a scalar model in the upper critical dimension, where all the conventional expansions can be compared with direct calculations, we claim that the field-theoretical result concerning the leading singularity of the two-point function at zero momentum given in Eq. (4.2) can be reproduced very well by the high-temperature expansion.

Using a parametrization of the subleading singularities depending on a single constant $r$, we obtained an optimal agreement for the choice $\gamma=0.9997$ and $p=0.3351$. With this choice, the error on $(h(m))^{-1}$ defined in section IV, is less than one part in a million for $300 \leq m \leq 400$. The small discrepancies between our estimate of the critical exponents and the field-theoretical values $\gamma=1$ and $p=1 / 3$ are not significant because it is possible to show that small changes in the exponents and allowing $r$ to slowly vary have comparable effects for the quality of the fit.

The present study shows that the use of asymptotic analysis or the use of a short series can be misleading. Given the length of the series available, asymptotic analysis may be useful for order of magnitude estimates but not for an accurate determination of the exponents.

There is still room for improvement. One could use calculations at fixed $\beta$ to study the corrections to the parametrization of Eq. (4.6). This procedure could be pursued up to the point where the main source of errors would be the numerical errors on the coefficient.

The use of the high-temperature expansion allows us to probe global features of the renormalization group flows which cannot be approached using renormalized perturbation theory or an analysis of the linearized behavior near the fixed point. In particular, our analysis makes implausible, for the model considered here, unconventional possibilities such as the existence in the upper critical dimension of a non-trivial fixed point characterized by non-trivial exponents. 


\section{ACKNOWLEDGMENTS}

This research was supported in part by the Department of Energy under Contract No. FG02-91ER40664. J.J. Godina is supported by a fellowship from CONACYT. 


\section{REFERENCES}

* At CERN until December 311997.

[1] K. Wilson, Phys. Rev. B. 43185 (1971) ; Phys. Rev. D. 31818 (1971).

[2] G. Baker, Phys. Rev. B 15, 1552 (1977).

[3] D. Gaunt, M. Sykes and S. McKenzie, J. Phys. A 12, 871 (1979).

[4] Y. Meurice, G. Ordaz and V. G. J. Rodgers, J. Stat. Phys. 77, 607 (1994).

[5] E. Brezin, J. C. Le Guillou and J. Zinn-Justin, in Phase Transitions and Critical Phenomena vol. 6, C. Domb and M. S. Green, eds., (Academic Press, New York, 1976).

[6] There is a large amount of literature on this subject. References can be found, e.g., in Phase Transitions, Cargese 1980, M. Levy, J.C. Le Guillou and J. Zinn-Justin eds., (Plenum Press, New York, 1982).

[7] G. Baker and N. Kawashima, Phys. Rev. Lett. bf 75, 994 (1995).

[8] F. Dyson, Comm. Math. Phys. 12, 91 (1969) ; G. Baker, Phys. Rev. B5, 2622 (1972).

[9] P. Bleher and Y. Sinai, Comm. Math. Phys. 45, 247 (1975) ; P. Collet and J. P. Eckmann, Comm. Math. Phys. 55, 67 (1977) and Lecture Notes in Physics 74 (1978) ; H. Koch and P. Wittwer, Comm. Math. Phys. 106495 (1986) , 138 (1991) 537 , 164 (1994) 627 .

[10] P. Collet, J.-P. Eckmann, and B. Hirsbrunner, Phys. Lett. 71B, 385 (1977).

[11] Y. Meurice and G. Ordaz, J. Stat. Phys. 82, 343 (1996).

[12] Y. Meurice and G. Ordaz, J. Phys. A 29, L635 (1996).

[13] Y. Meurice, G. Ordaz and V. G. J. Rodgers, Phys. Rev. Lett. 75, 4555 (1995) .

[14] Y. Meurice, S. Niermann and G. Ordaz, J. Stat. Phys. 87, 363 (1997).

[15] B. Nickel, Lecture Notes published in Ref. [6].

[16] Th. Niemeijer and J. van Leeuwen, in Phase Transitions and Critical Phenomena, vol. 6, C. Domb and M. S. Green, eds., (Academic Press, New York, 1976).

[17] J.J. Godina, Y. Meurice, and S. Niermann, Univ. of Iowa preprint 97-2501, CERN 97-251, hep-lat 9709097.

[18] A. J. Guttmann, in Phase Transitions and Critical Phenomena vol. 13, C. Domb and Lebowitz, eds., (Academic Press, New York, 1989).

[19] G. Parisi, Statistical Field Theory (Addison Wesley, New-York, 1988). 559 (1983); 


\section{FIGURES}

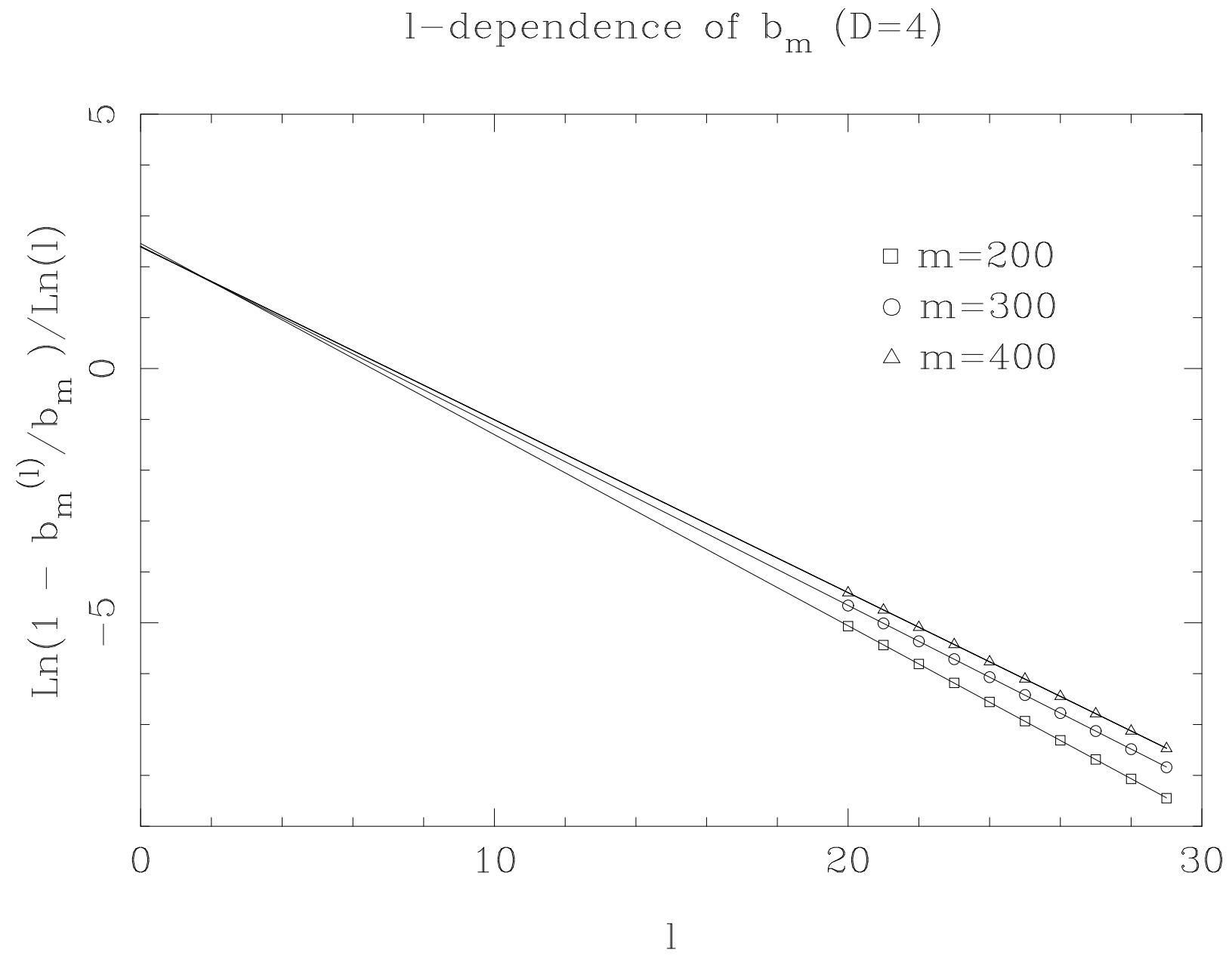

FIG. 1. $l$-dependence of the high-temperature coefficients $b_{m}^{(l)}$ calculated in truncated spaces of dimension $l$. 


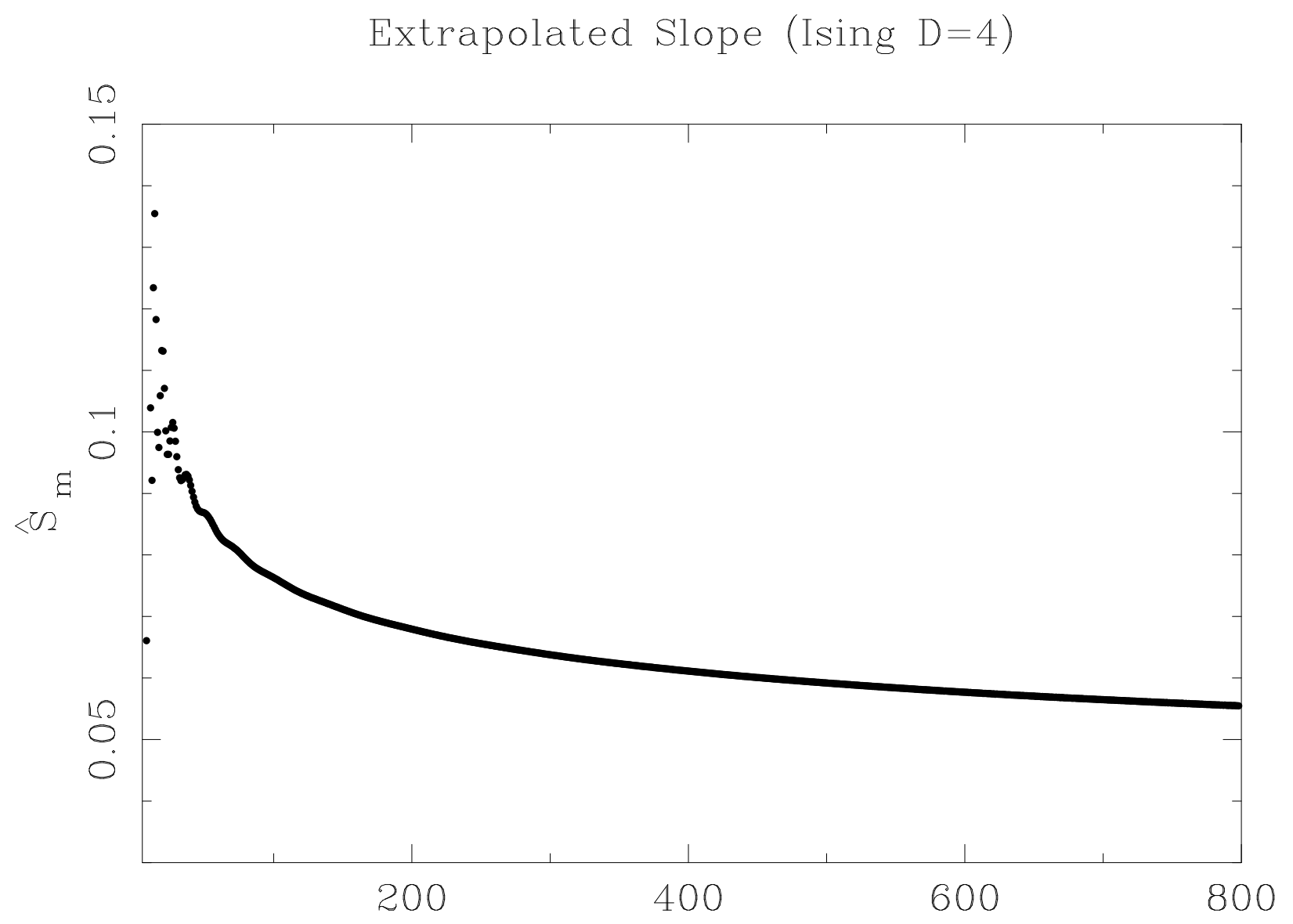

In

FIG. 2. The extrapolated slope $\widehat{S}_{m}$ versus the order $m$ in the HT expansion. 
Inverse Extrapolated Slope (Ising D=4)

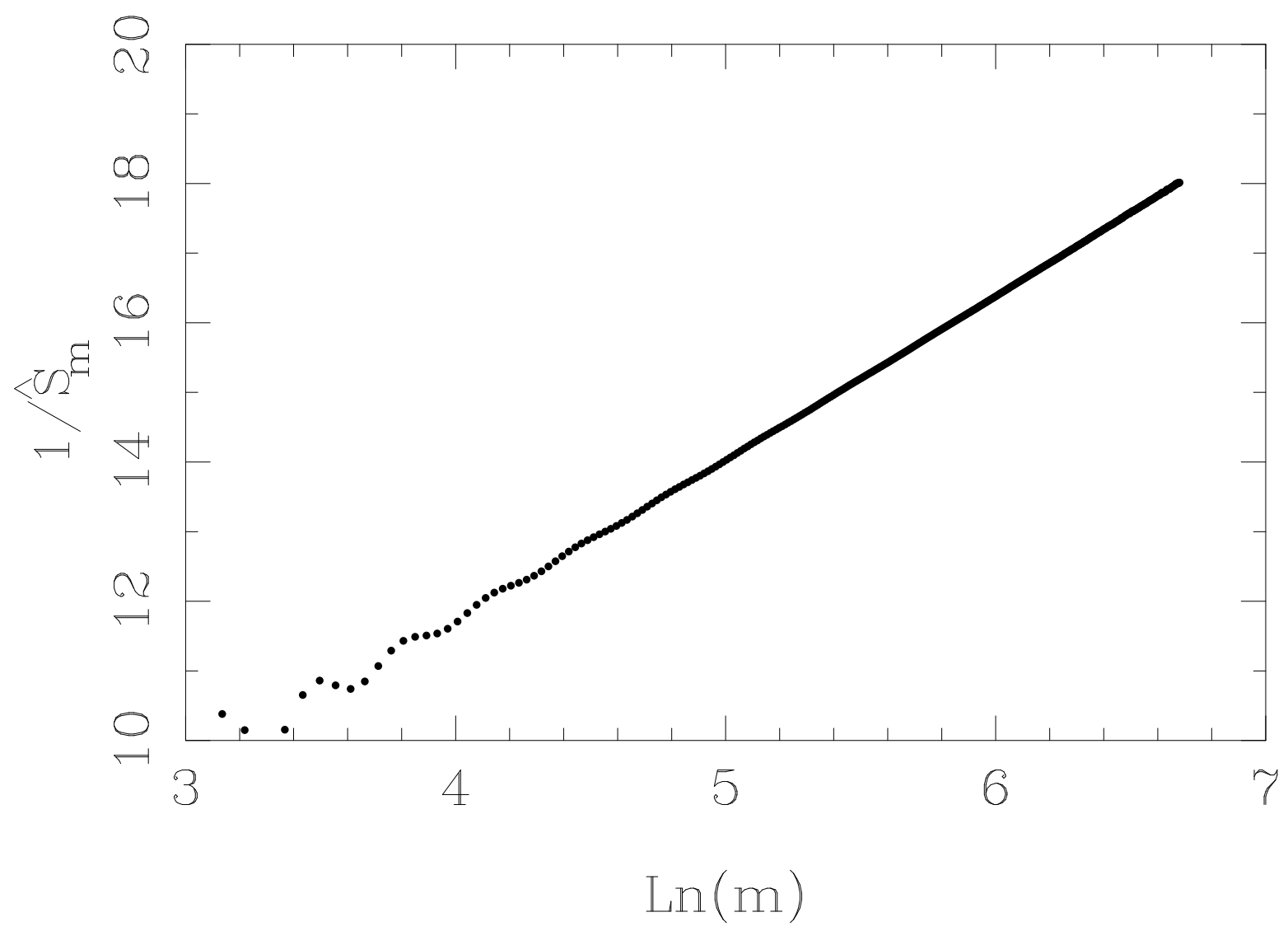

FIG. 3. The inverse extrapolated slope $\widehat{S}_{m}$ versus the order $m$ in the HT. 


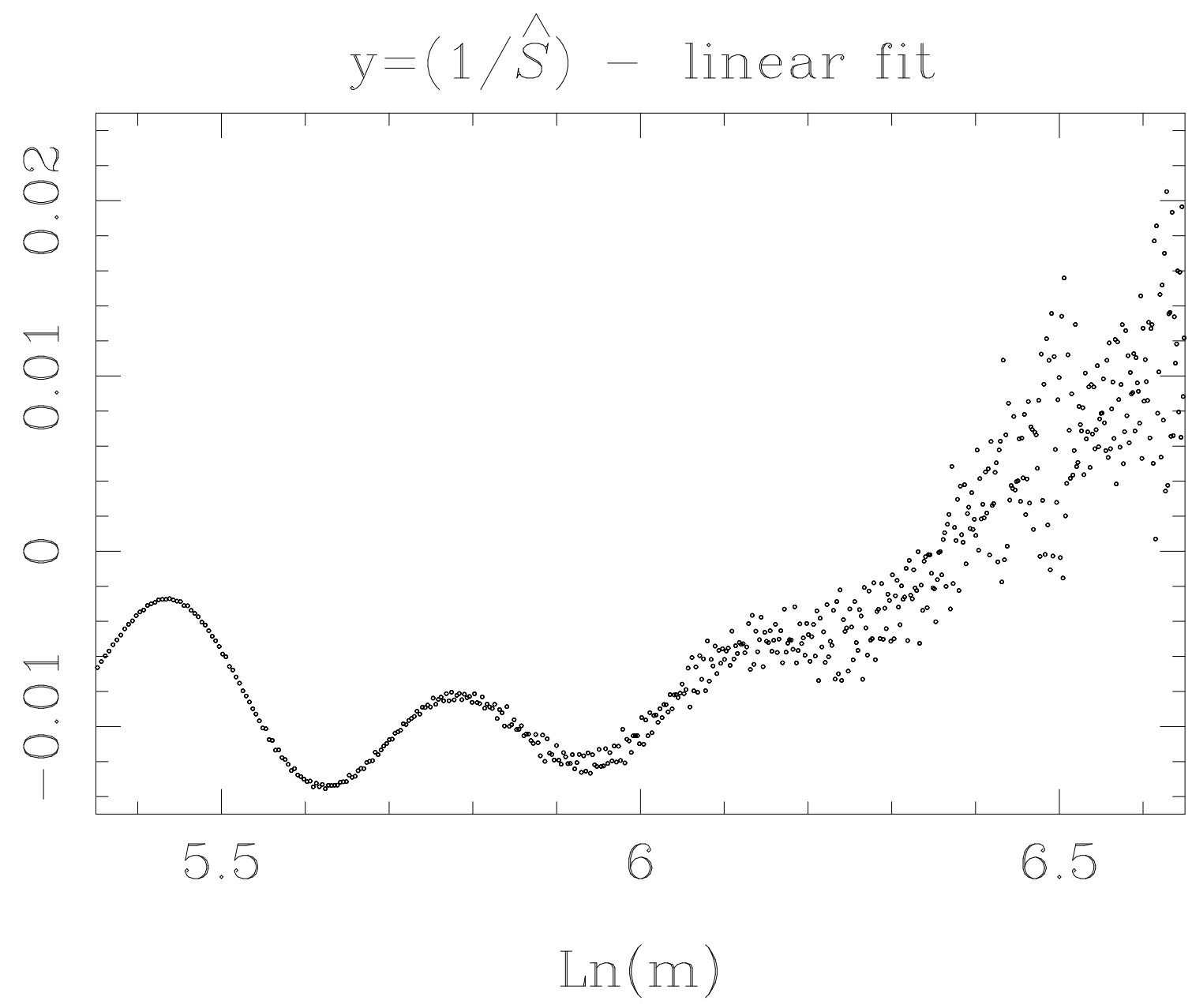

FIG. 4. Difference between $\widehat{S}_{m}^{-1}$ and a linear fit in $\operatorname{Ln}(m)$. 


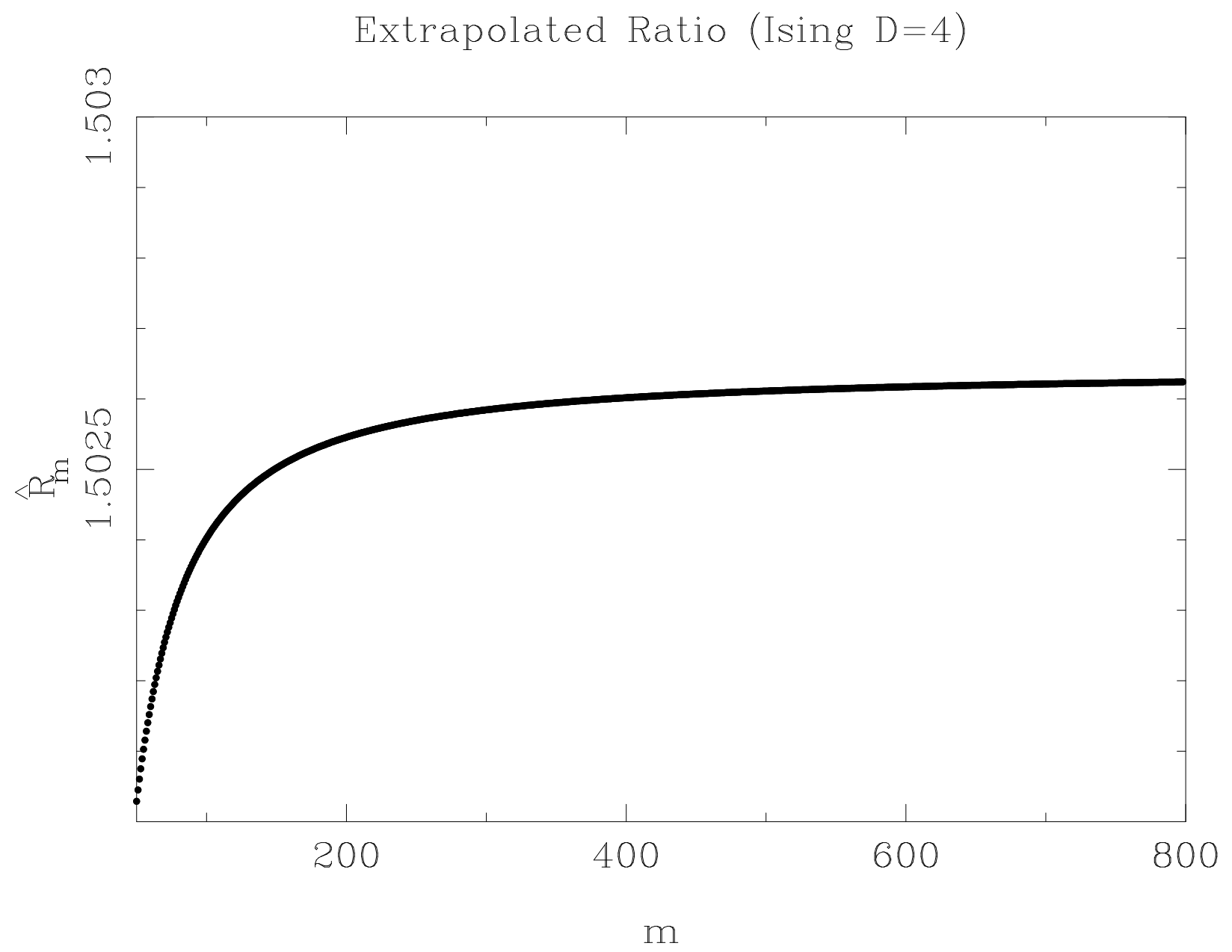

FIG. 5. The extrapolated ratio $\widehat{R}_{m}$ versus the order $m$ in the HT expansion. 


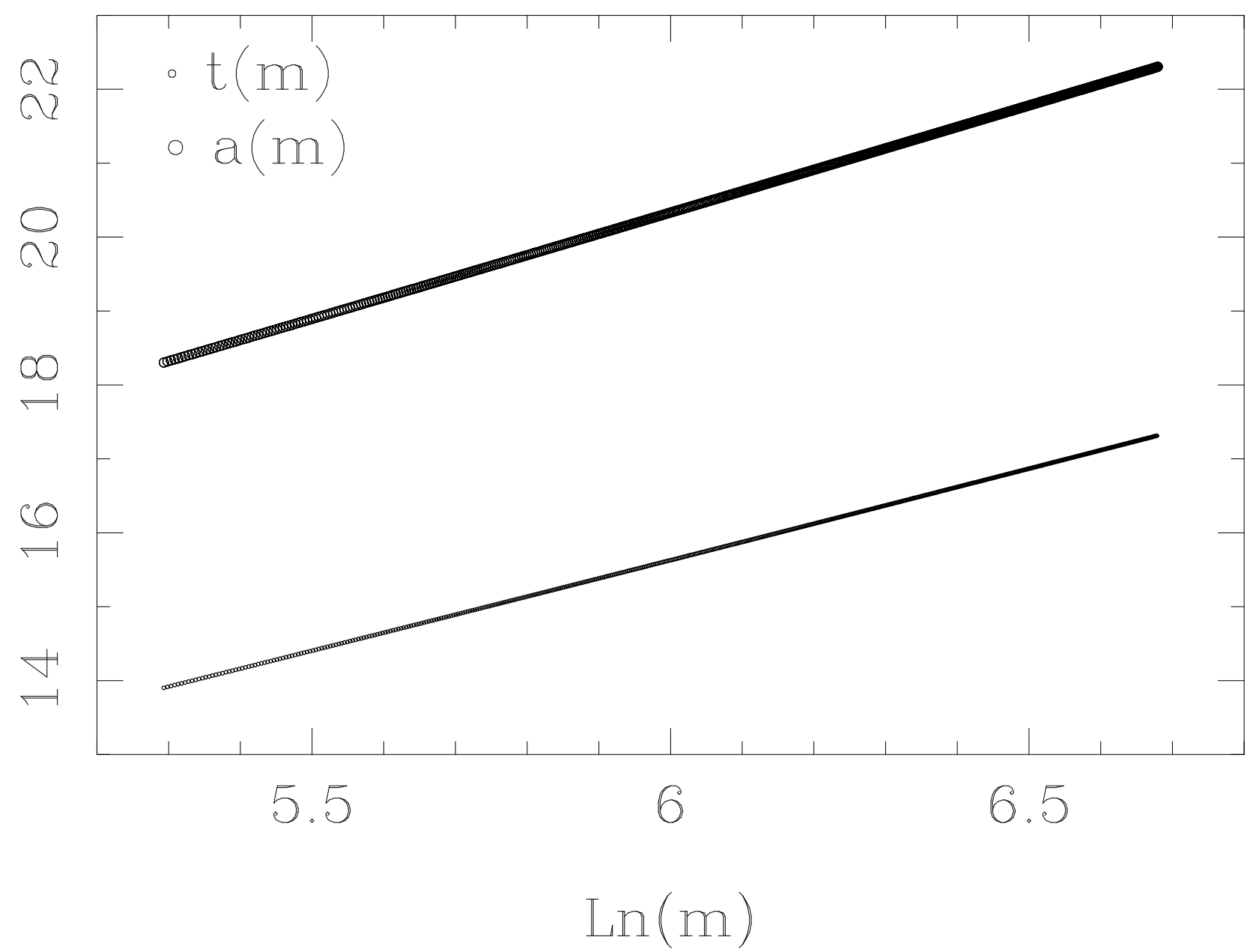

FIG. 6. $(h(m))^{-1}$ versus $\operatorname{Ln}(m)$ from Eq. (4.3) (large circles, above) and from the actual HT series (small circles, below). 


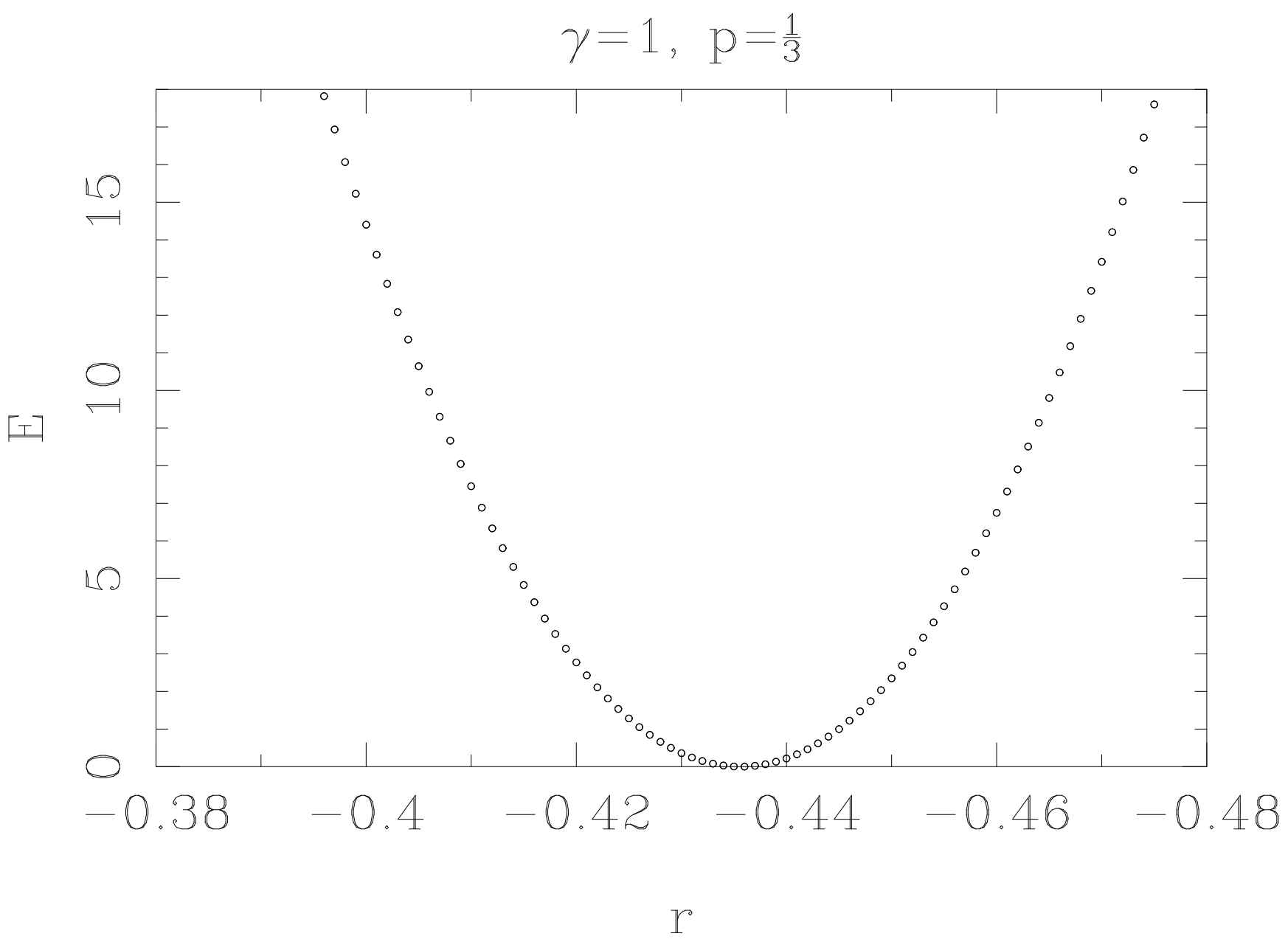

FIG. 7. The function $E$ defined in Eq. (4.5) versus $r$, with $a(m)$ calculated from Eq. (4.4) 


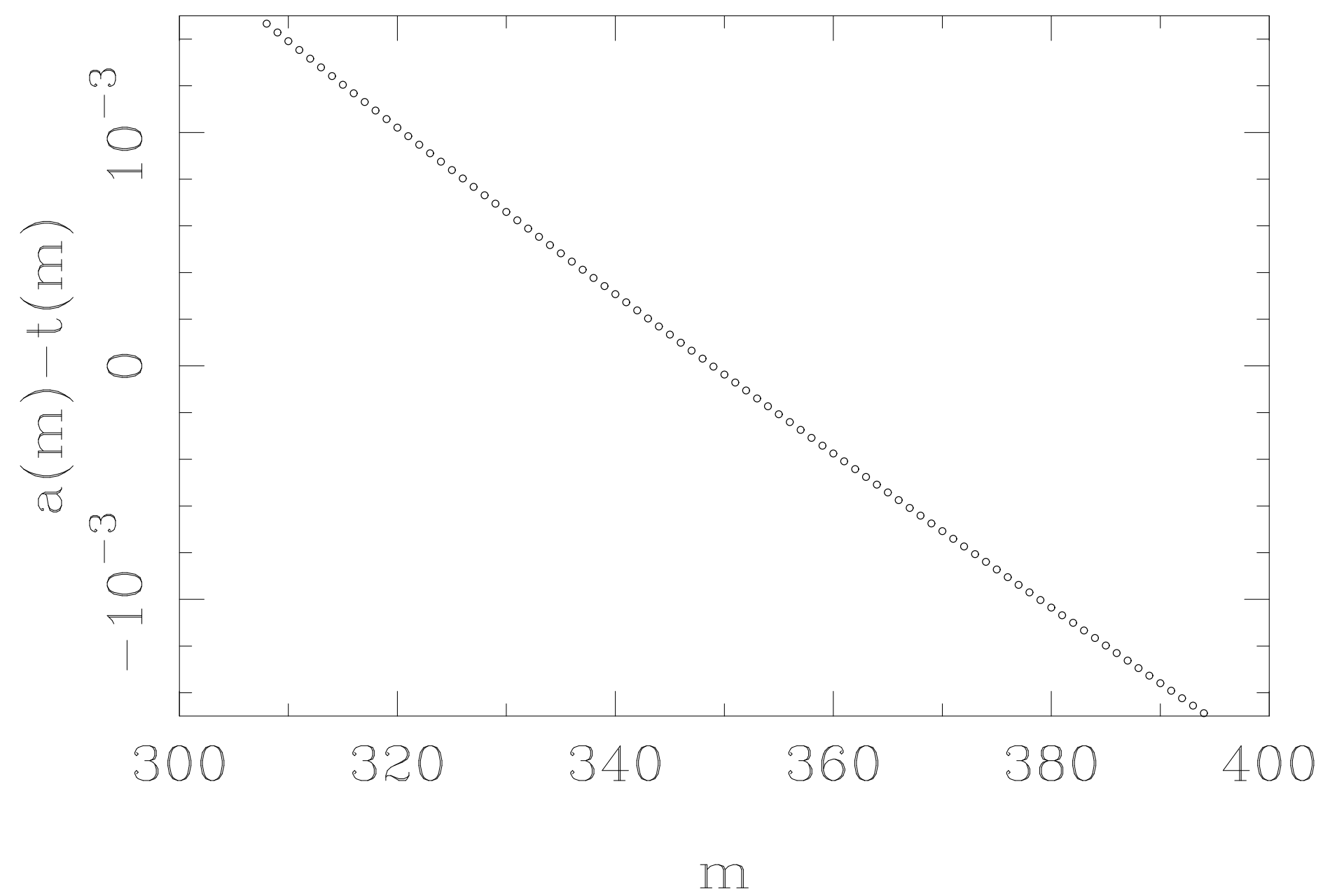

FIG. 8. $a(m)-t(m)$ versus $m$ for $a(m)$ calculated with $r=-0.435622$ in Eq.(4.4) 


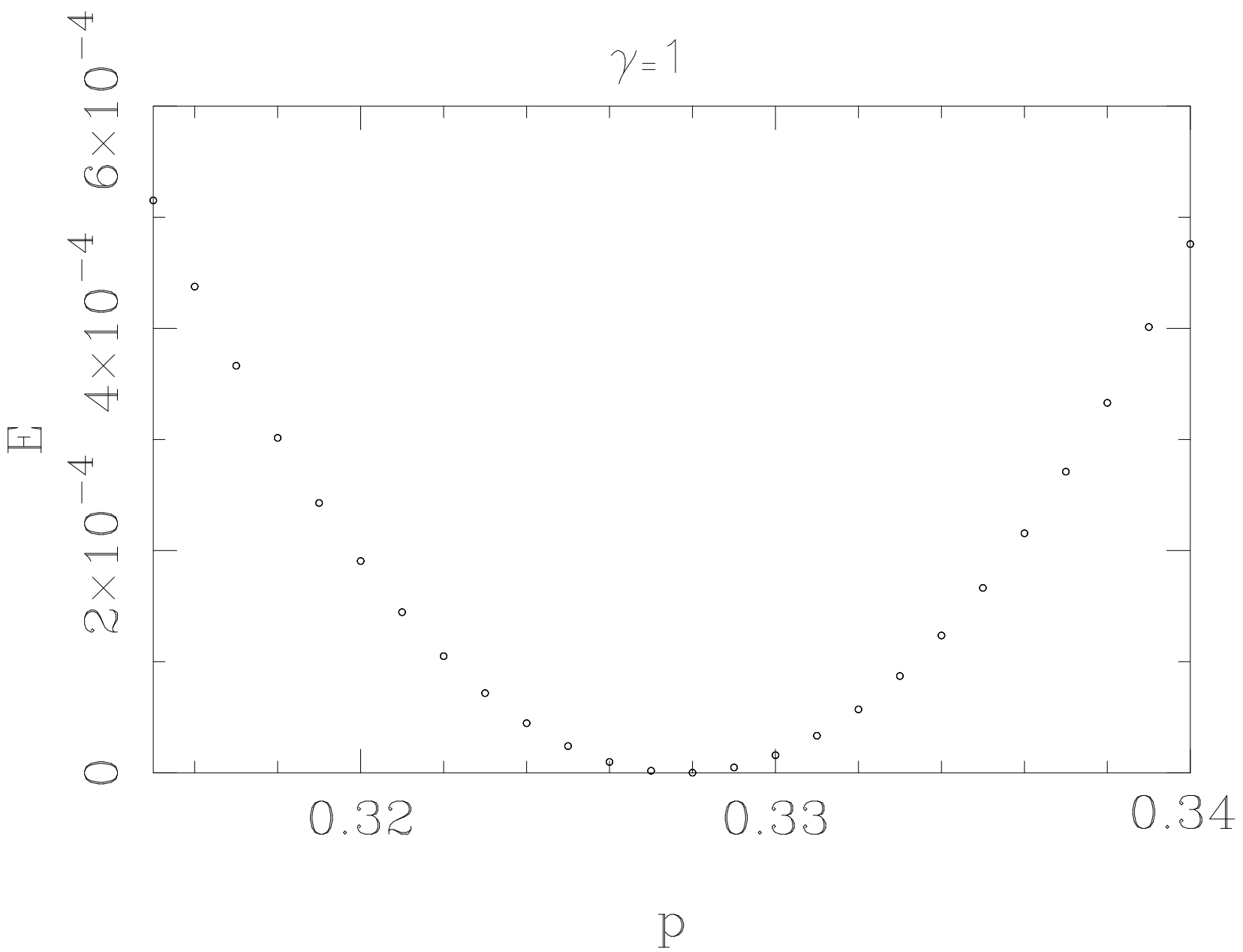

FIG. 9. The function $E$ versus $p$ with $\gamma=1$ and $r$ chosen to minimize $E$. 


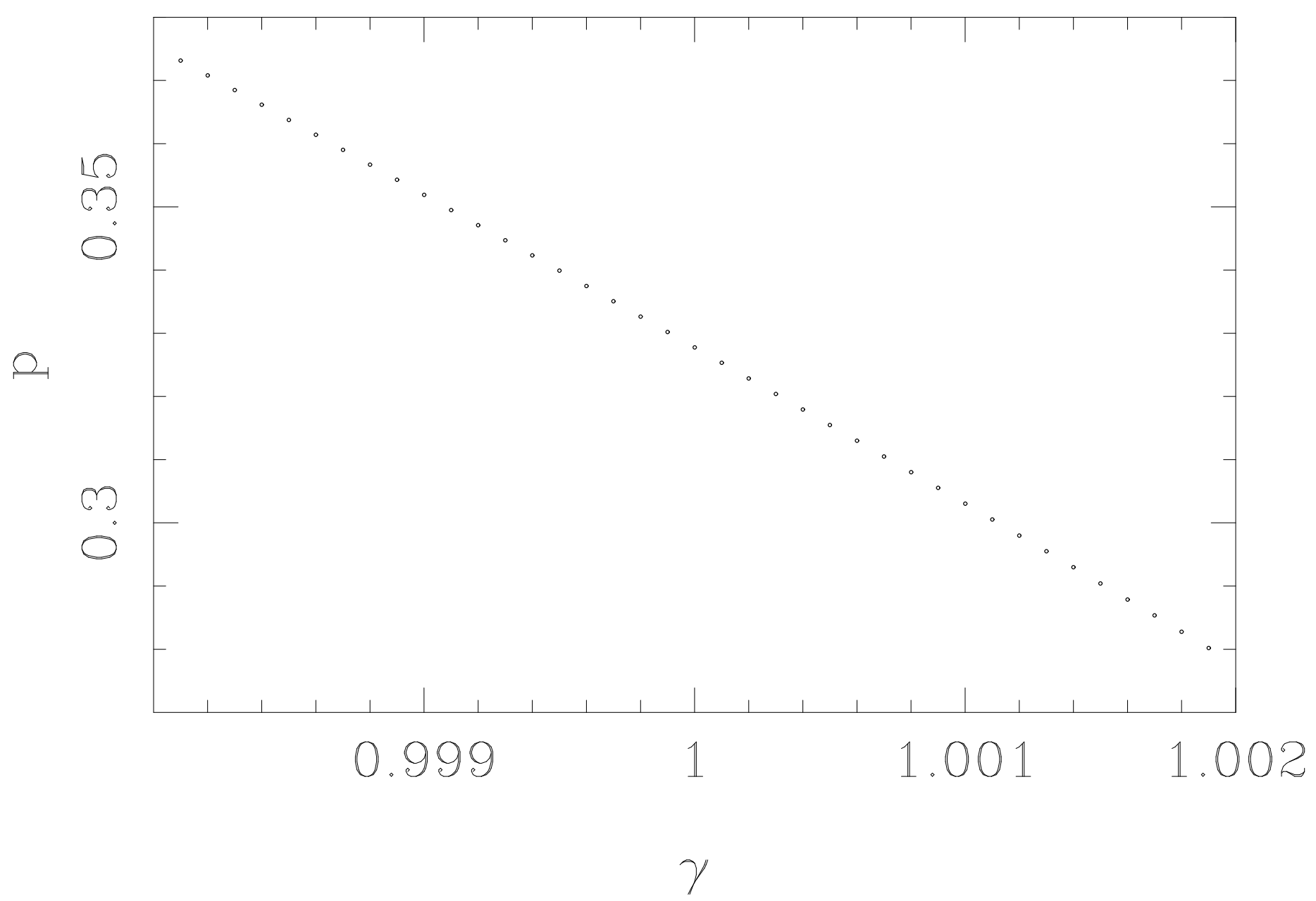

FIG. 10. Values of $p$ minimizing $E$ at given $\gamma$ and optimum $r$. 


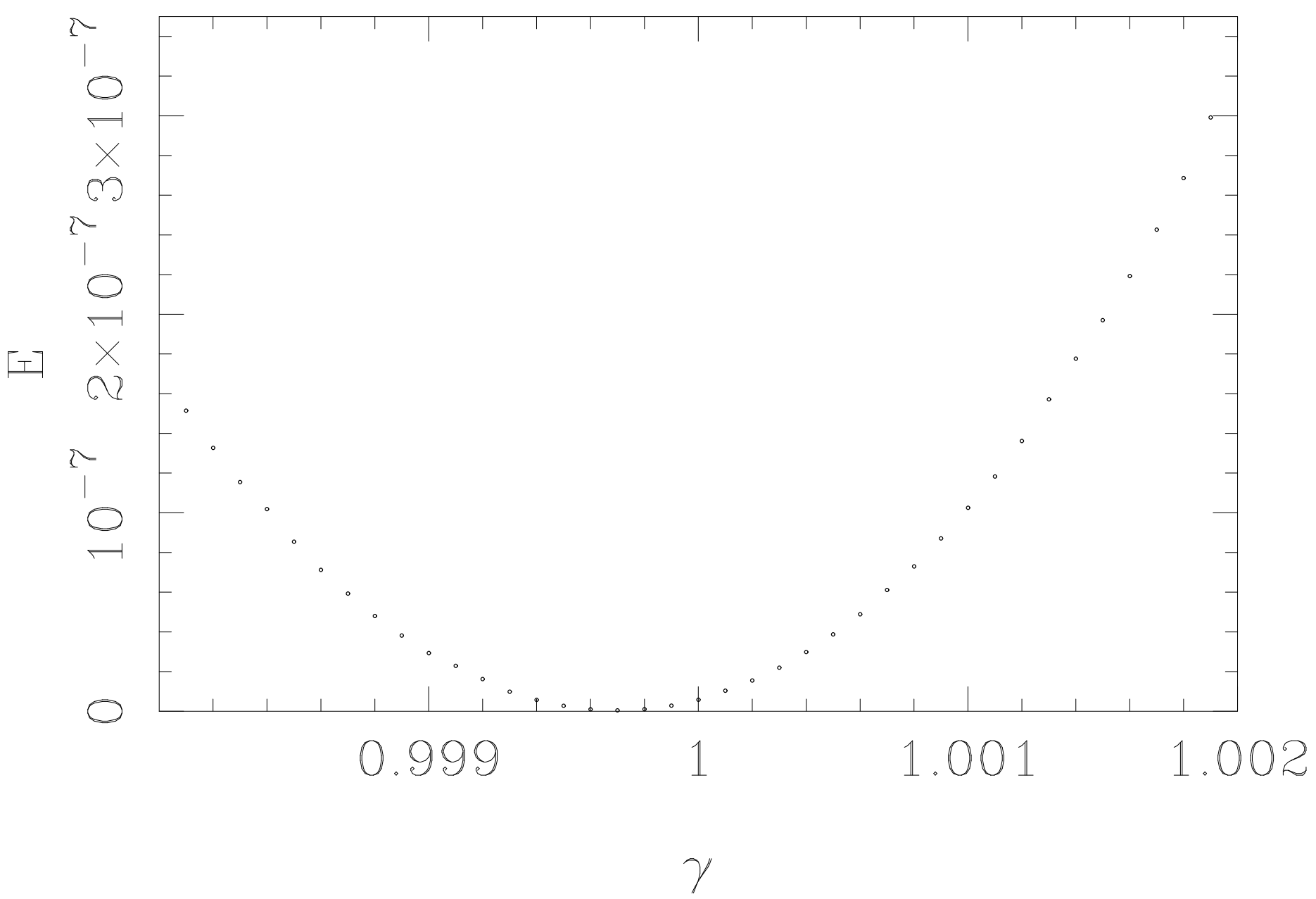

FIG. 11. The function $E$ versus $\gamma$ with values of $p$ and $r$ chosen to minimize $E$. 


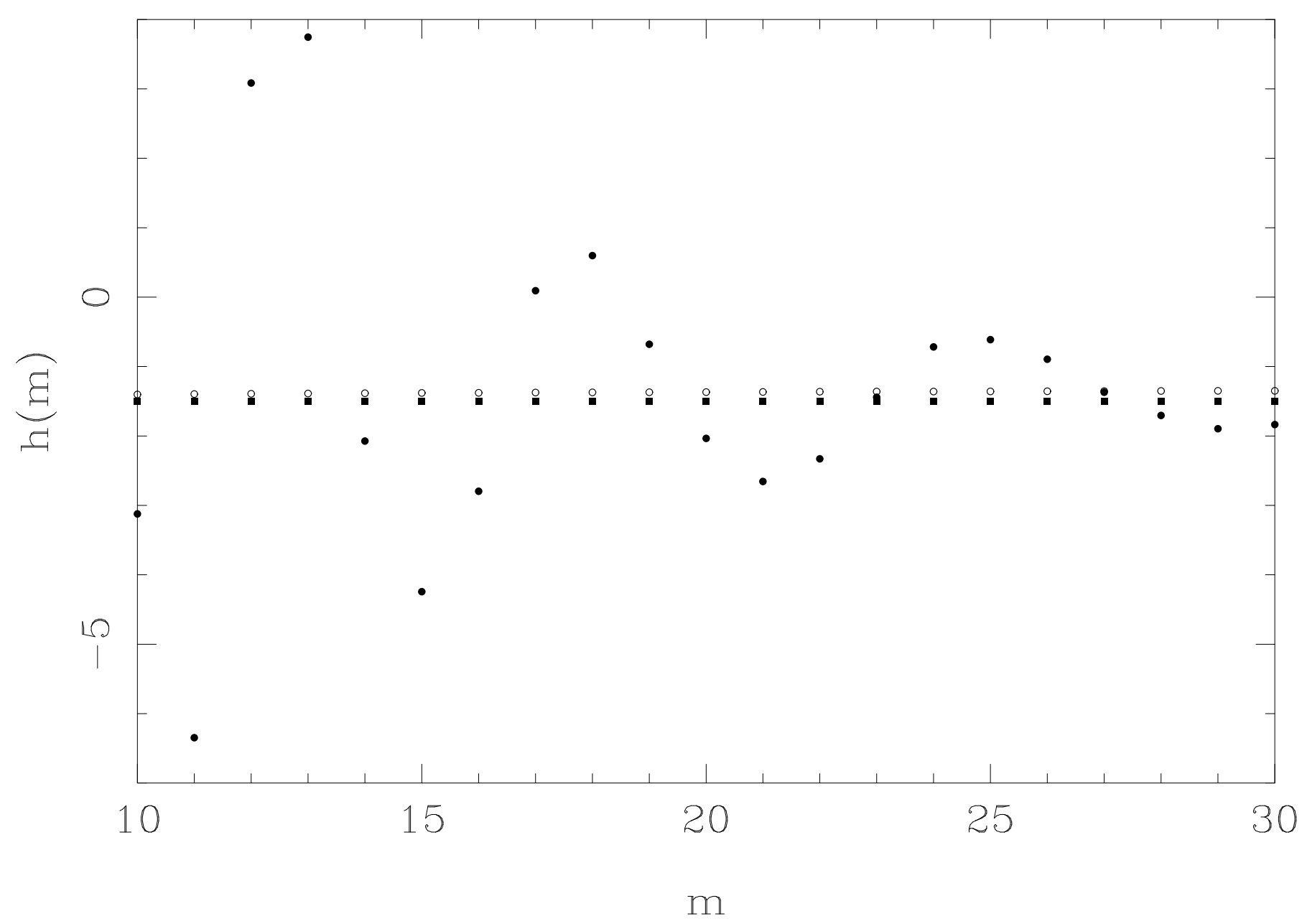

FIG. 12. The function $h(m)$ corresponding to the HT expansion of $l a m b d a_{4}$ (dots) compared to the same function for $-x / \operatorname{Ln}(1-x)$ (circles) and $(1-x)^{\frac{1}{2}}$ (squares). 


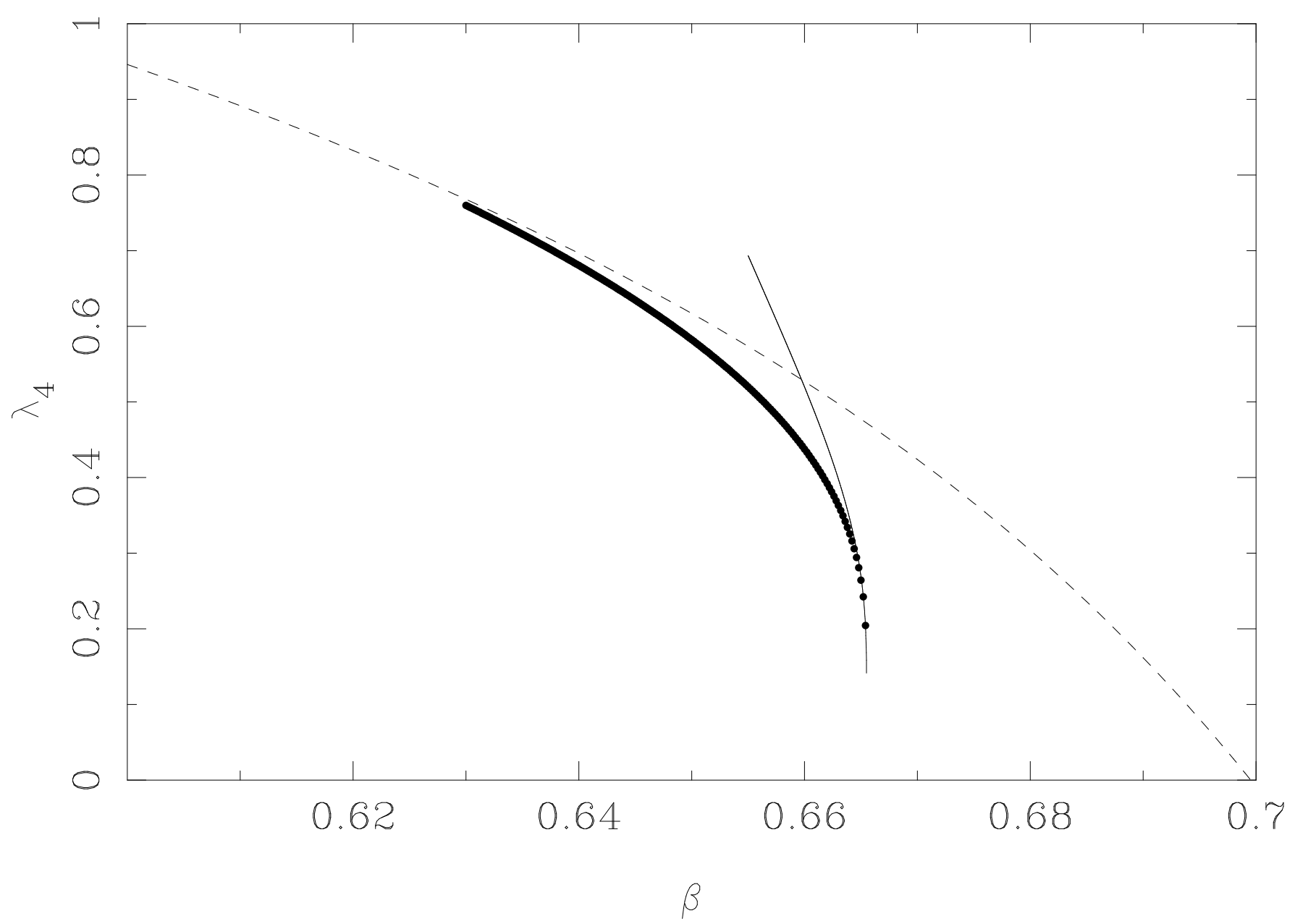

FIG. 13. $\lambda_{4}$ versus $\beta$, exact (dots), with the HT expansion up to order 30 (dashed) and in leading singularity (line). 